\title{
¿Son confiables las fotografías para el análisis facial en ortodoncia?
}

\section{Are photographs for facial analysis in orthodontics reliable?}

\author{
María Paz Astudillo-Loyola ${ }^{1}$, Shadi Dehghan-Manshadi-Kemm² ${ }^{\star}$,Cristian Vergara-Nuñez ${ }^{3}$, \\ Cristian Peñafiel-Ekdhal ${ }^{4}$
}

\author{
1. Cirujano Dentista, Facultad de Odontología \\ Universidad de Chile, Chile. \\ 2. Práctica privada \\ 3. Cirujano Dentista, Facultad de Odontología \\ Universidad de Chile, Chile. \\ 4. Tecnólogo Médico, Facultad de Odontología \\ Universidad de Chile, Chile.
}

* Correspondencia autor: Shadi Dehghan-

Manshadi-Kemm

Dirección: La Concepción 81 of. 1604, Providencia. Santiago, Chile. | Teléfono: (+56 9) 84797621 |

E-mail: shadimanshadi@gmail.com

Trabajo recibido el 07/09/2017. Aprobado para su publicación el 07/01/2018

\section{RESUMEN}

Introducción: Antropometría facial es la medición de distancias del rostro. Un método indirecto es a través de fotografías estandarizadas, conocido como fotogrametría. Este trabajo busca estandarizar una técnica fotográfica y estudiar la fiabilidad de la fotogrametría facial, comparando mediciones antropométricas directas y fotogramétricas con tres objetivos fotográficos distintos. Objetivo: Estandarizar parámetros de la cámara fotográfica y encuadre de la imagen para fotogrametría facial. Materiales y métodos: A una muestra de 50 sujetos se les midieron directa e indirectamente seis distancias faciales. Para las fotografías se utilizaron objetivos fotográficos fijos de $35 \mathrm{~mm}, 50 \mathrm{~mm}$ y $100 \mathrm{~mm}$. Se tomaron seis fotografías a cada uno de los voluntarios; tres de frente y tres de perfil, una con cada lente. La distancia entre la cámara y el sujeto fue variable, siempre con un encuadre de $30 \mathrm{~cm}$. Se compararon las diferencias estadísticamente. Resultados: Con el lente de $100 \mathrm{~mm}$ fue el único con el que todas las distancias medidas en fotografías fueron iguales y sin diferencia estadística respecto a las medidas obtenidas directamente. Conclusión: De los tres objetivos fotográficos utilizados uno de $100 \mathrm{~mm}$ es el que permite la fotografía más fiel a la realidad, con una diferencia promedio de $0,5 \mathrm{~mm}$ con las medidas directas.

PALABRAS CLAVE

Fotogrametría, Antropometría facial indirecta, Fotografías estandarizadas.

Rev. Clin. Periodoncia Implantol. Rehabil. Oral Vol. 11(1); 13-15, 2018.

\section{ABSTRACT}

Introduction: Facial anthropometry is the measurement of distances of the face. It can be performed directly in the subject or indirectly. Among the indirect methods is photogrammetry: measuring facial distances in photographs. In order to perform the facial photographic registration, a standardized photographic technique is required, that is, the parameters of the camera, the photographic set and the parameters of the subject must be previously established. This work aims to standardize a photographic technique and to study the reliability of facial photogrammetry, comparing direct anthropometric measurements and photogrammetry with three different photographic lenses, in order to determine which technique has the highest fidelity. Objective: To standardize camera parameters and image framing for facial photogrammetry. Materials and Methods: Six facial distances were measured directly and indirectly on a sample of 50 subjects. The indirect measurements were taken on photographs of the subjects using fixed photographic lenses of $35 \mathrm{~mm}, 50 \mathrm{~mm}$ and $100 \mathrm{~mm}$. Six photographs were taken from each volunteer; three front and three profile photographs, one with each lens. The distance between the camera and the subject was variable, so that the image frame was always $30 \mathrm{~cm}$. The differences were statistically compared. Results: With a $35 \mathrm{~mm}$ lens, only one of the six distances measured in photographs was equal to the measurement obtained directly. With a $50 \mathrm{~mm}$ lens, three of the six distances obtained were equal to the direct measurements. With a $100 \mathrm{~mm}$ lens, all distances measured in photographs were equal and without statistical difference in relation to the measurements obtained directly. Conclusion: Of the three photographic lenses, the $100 \mathrm{~mm}$ is the lens that gives the most faithful photograph to the reality, with a difference of $0,5 \mathrm{~mm}$ or less with the direct measurements.

\section{KEY WORDS}

Photogrammetry, Indirect facial anthropometry, Standardized photographs.

Rev. Clin. Periodoncia Implantol. Rehabil. Oral Vol. 11(1); 13-15, 2018. 


\section{HIPÓTESIS}

Las medidas faciales obtenidas mediante fotogrametría estandarizada son iguales a las obtenidas mediante antropometría directa.

\section{OBJETIVOS}

- Establecer parámetros de la cámara fotográfica y encuadre adecuado para fotogrametría facial estandarizada.

- Comparar las medidas obtenidas mediante fotogrametría con lente de $35 \mathrm{~mm}, 50 \mathrm{~mm}$ y $100 \mathrm{~mm}$ con las medidas antropométricas directas.

- Definir el o los lentes que proporcionan mayor fidelidad a la técnica fotográfica.

\section{MATERIALES Y MÉTODOS}

El presente estudio es de tipo observacional analítico. La selección de la muestra fue por conveniencia, no probabilístico. Los voluntarios corresponden a estudiantes de $1^{\circ}$ a $6^{\circ}$ año de la Facultad de Odontología de la Universidad de Chile, entre los años 2016 - 2017. Fueron incluidos en 30 mujeres y 20 hombres, adultos jóvenes entre 15 y 29 años de edad, y que aceptaran la participación en el estudio mediante la firma de un consentimiento informado aprobado por el Comité de Ética Científico local. Como criterio de exclusión se consideró el tener alguna discapacidad motora que les impidiera mantener una postura de pie erguida y estable. A cada voluntario se le explicó de manera verbal y escrita en qué consistía su participación en el estudio.

\section{Antropometría directa}

Se midieron seis distancias a cada sujeto, tres de frente y tres de perfil. Las mediciones se realizaron con un compás de punta seca y una regla metálica milimetrada de $30 \mathrm{~cm}$. Las mediciones se registraron en una planilla Excel para su posterior análisis. Las distancias estudiadas son presentadas en la figura 1.

Si un voluntario presentaba incompetencia labial se utilizó como referencia el punto Stomion superior en vez de Stomion.

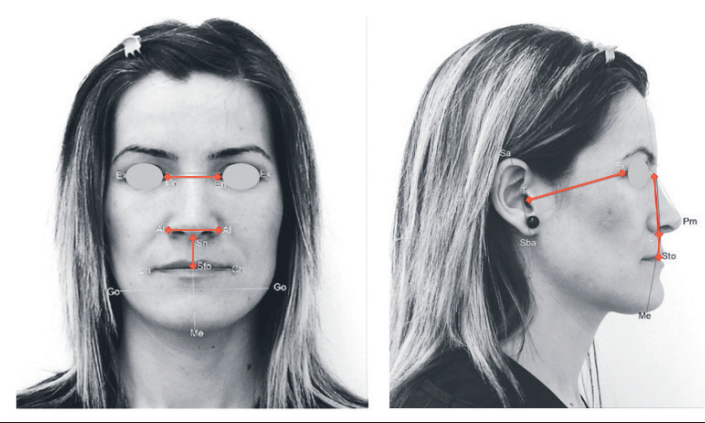

Figura 1. Distancias faciales Imagen obtenida de "Reliability of reference distances used in photogrammetry." (Aksu y cols., 2010). Distancias faciales frontales: En-En Ancho intercantal, Al-Al Ancho alar, Sn-Sto Largo labio superior. Distancias faciales de perfil: Tr-Ex Distancia orbitotragal, Na-Sn Altura nasal, SnSto(2) Largo labio superior.

\section{Antropometría indirecta}

Se tomaron seis fotografías a cada sujeto, una de frente y una de perfil con tres objetivos fotográficos distintos (35mm, $50 \mathrm{~mm}$ y $100 \mathrm{~mm}$ ). Se estandarizó un encuadre de $30 \mathrm{~cm}$. en cada fotografía, medido con una regla de madera posicionada a un lado del voluntario. La distancia cámara-sujeto fue determinada por el encuadre especificado y la distancia del sujeto al fondo fue de $30 \mathrm{~cm}$. La postura del voluntario al momento de la fotografía debía ser en Posición Natural de Cabeza (PNC), descrito por Solow y Tallgren en $1971^{(6,7,8)}$, tanto para fotografías de frente como de perfil.

Se utilizó una cámara réflex digital Pentax K-3 programada en manual, con velocidad de obturación $1 / 125$, apertura de diafragma $\mathrm{f}=11$, sensibilidad a la luz ISO 100 y con un flash Pentax AF540FGZII programado en manual con difusor. La cámara fue sostenida por un trípode Gitzo Serie 3 con un cabezal Gimbal Wimberley WH-200 para asegurar la inmovilidad del equipo. El punto de enfoque para fotografías frontales fue el punto nasion y para fotografías de perfil fue el ala nasal.

Se realizaron las mediciones de las seis distancias estudiadas en el sujeto y las fotografías con los tres lentes. Para las mediciones de las fotos digitales se utilizó el software UTHSCSA ImageTool 3.0. Cada medición se realizó tres veces y se obtuvo un promedio.

\section{Análisis estadístico}

Se utilizó el software STATA 14 S/E ${ }^{\circledR}$ para el análisis estadístico. El test Shapiro-Wilk demostró que la mayoría de los datos tenían distribución normal y una serie fue no normal. Para comparar las medidas antropométricas directas con las indirectas se utilizaron las pruebas de Wilcoxon y T-Test según su distribución estadística.

\section{RESULTADOS}

Se compararon las medidas obtenidas con antropometría directa, con las obtenidas mediante fotogrametría con cada uno de los lentes utilizados. Los resultados se muestran en la tabla 1.

Tabla 1. Comparación entre mediciones antropométricas y fotogramétricas.

\begin{tabular}{c|c|c|c|c}
\hline Medida & $\begin{array}{c}\text { Lente } \\
\mathbf{3 5 m m}\end{array}$ & $\begin{array}{c}\text { Lente } \\
\mathbf{5 0 m m}\end{array}$ & $\begin{array}{c}\text { Lente } \\
\mathbf{1 0 0} \mathbf{m m}\end{array}$ & Test utilizado \\
\hline En-En & $p=0.00$ & $p=0.06$ & $p=0.56$ & Test de Wilcoxon \\
Al-Al & $p=0.00$ & $p=0.00$ & $p=0.14$ & Test de Wilcoxon \\
Sn-Sto & $p=0.00$ & $p=0.02$ & $p=0.39$ & Test de Wilcoxon \\
Tr-Ex & $p=0.00$ & $p=0.00$ & $p=0.47$ & T - test \\
Na-Sn & $p=0.72$ & $p=0.13$ & $p=0.06$ & Test de Wilcoxon \\
Sn-Sto (2) & $p=0.03$ & $p=0.28$ & $p=0.96$ & Test de Wilcoxon \\
\hline
\end{tabular}

Las medidas en rojo son aquellas que no presentaron diferencia estadística al medirlas de forma directa e indirecta.

Las distancias medidas en fotografías obtenidas con lente de $35 \mathrm{~mm}$ fueron diferentes a las obtenidas directamente, a excepción de la distancia $\mathrm{Na}-\mathrm{Sn}$. Con lente de $50 \mathrm{~mm}$ tres de las 6 distancias no presentaron diferencia estadística con las medidas directas; la distancia En - En, $\mathrm{Na}-$ $\mathrm{Sn}$ y $\mathrm{Sn}-\mathrm{Sto}^{(9,10)}$. Con el lente de $100 \mathrm{~mm}$ todas las distancias estudiadas resultaron muy similares a las obtenidas de forma directa, sin diferencia estadística entre ellas.

Luego se calculó el promedio de cada una de las distancias medidas de forma directa y con cada uno de los lentes.

Con lente de $35 \mathrm{~mm}$ se obtuvo en promedio una diferencia de $2,7 \mathrm{~mm}$ entre medidas directas e indirectas. Con el lente de $50 \mathrm{~mm}$ se obtuvo una diferencia promedio de $0,9 \mathrm{~mm}$ entre las distancias directas e indirectas sin diferencia estadística y de $2,2 \mathrm{~mm}$ en las distancias que presentaron diferencia estadística. Con lente de $100 \mathrm{~mm}$ se logró obtener una diferencia promedio de $0,5 \mathrm{~mm}$ entre las distancias medidas de forma directa e indirecta, siendo la diferencia mínima de $0 \mathrm{~mm}$ y la máxima de $1,3 \mathrm{~mm}$.

\section{DISCUSIÓN}

El uso de la fotografía digital es cada vez más común dentro de las áreas médicas, ya que se utiliza como medio de registro para diferentes fines. Sus grandes ventajas recaen en su rapidez, facilidad de obtención y bajo costo, sin olvidar su invariabilidad en el tiempo.

A pesar de sus ventajas, existen dudas sobre la reproducción de los elementos que se registran mediante una fotografía, es decir, qué tan parecidas a la realidad son las imágenes obtenidas. Existen publicaciones sobre la técnica fotográfica, recomendaciones de lentes que se deben ocupar según lo que se quiere fotografiar, posición del sujeto, etc., pero hay pocos trabajos que se hayan dedicado a comparar las distancias obtenidas mediante fotografía facial y las mismas medidas tomadas directamente en el paciente. Dentro de estos trabajos se encuentran algunos que comparan antropometría con fotogrametría facial. La fotogrametría facial se ha reportado como un método de antropometría indirecta preciso, simple y económico ${ }^{(1)}$

De los artículos publicados respecto a la comparación de antropometría facial directa con fotogrametría facial en 2D, destaca el trabajo de Farkas y cols., $1980^{(3)}$, dónde se compararon distancias, ángulos e inclinaciones faciales. Como resultado se obtuvo que un $41.9 \%$ de las medidas estudiadas eran confiables en la fotografía, principalmente las que correspondían a inclinaciones faciales. Otro estudio es el realizado por Han y cols. el $2010^{(1)}$, quien también comparó distancias lineales, ángulos e inclinaciones faciales, sin embargo, sus resultados mostraron mayores similitudes entre las mediciones, principalmente en las distancias lineales. Las diferencias entre estos trabajos puede deberse por una parte al avance tecnológico que ha experimentado la fotografía, pasando de lo análogo a lo digital, tanto en la parte fotográfica como computacional, y por otra parte a las diferencias metodológicas utilizadas (diferentes lentes, posición del sujeto, distancia del sujeto y la cámara, etc.). 
Un tercer estudio es el realizado por Aksu y cols. el 2010(11), quien midió solamente distancias lineales. Obtuvo resultados similares entre antropometría y fotogrametría en menos del $20 \%$ de las distancias estudiadas, lo que está muy por debajo de los trabajos expuestos anteriormente. Esta gran diferencia puede deberse al lente utilizado por Aksu (lente de $35 \mathrm{~mm}$ gran angular que genera distorsión en barril en las fotografías) y a la metodología del estudio, ya que para llevar la imagen a escala real utilizó una segunda distancia como referencia y dependiendo de la referencia utilizada sus resultados variaban.

En los estudios mencionados anteriormente se usó gran aparatología para la toma de fotografías, tales como cefalostatos, sillas modificadas para estandarizar enfoque y posición de cabeza, varios flash adicionales en diferentes posiciones alrededor del sujeto y paraguas reflectores, entre otros.

En relación al protocolo fotográfico, en este trabajo se modificó en diversos aspectos, tales como configuración de la cámara, iluminación, posición del individuo, distancia cámara-sujeto, todo esto buscando simplicidad a los protocolos descritos en los trabajos anteriores.

Respecto a la distancia cámara-sujeto, se decidió estandarizar cada fotografía a un encuadre de $30 \mathrm{~cm}$, necesario para que apareciera en la imagen la cabeza y cuello, además de la regla para medir el encuadre y posteriormente llevar la fotografía a escala real. La distancia cámarasujeto varió con cada lente utilizado, sin embargo, todas las distancias necesarias para generar el encuadre definido se encontraron dentro de las disponibles en una clínica dental promedio.

La posición del sujeto al momento de la fotografía también es algo que ha sido reportado de manera extensa. Bastantes autores afirman que la Posición Natural de Cabeza debe ser la posición inicial para fotografía debido a que otros parámetros basados en referencias intracraneales como el plano de Frankfurt, presentan gran variabilidad ${ }^{(12,13,14,15)}$. Es por eso que en este estudio se utilizó la PNC como posición del paciente para la fotografía, a diferencia de lo realizado por Han y cols., 2010(1), quienes usaron el plano de Frankfurt paralelo a la horizontal con asistencia de cefalostato y Farkas y cols., 1980 ${ }^{(3)}$, que utilizaron el plano de Frankfurt $5^{\circ}$ sobre la horizontal.

Se usó una cámara Reflex y no otra debido a las ventajas que ofrece en cuanto a la calidad de imagen al tener un mayor tamaño de sensor, lo que es importante para poder realizar distintos tipos de comparaciones. Otra ventaja es tener lentes u objetivos fotográficos intercambiables con longitud focal fija, configuración manual con diversas opciones, como por ejemplo usar un número f adecuado para tener una profundidad de campo correcta, regular la sensibilidad a la luz (ISO), la velocidad de obturación, la intensidad del flash, etc. y no presentar error de paralaje ${ }^{(16,17,18)}$.
Dentro de las limitaciones del estudio, se encuentra el número de sujetos examinados para tener resultados más concluyentes. Esto resultó difícil debido a la tarea de las mediciones antropométricas directas y sobre todo a la toma de fotografías con tres lentes distintos y a diferentes distancias, lo que implicó mover constantemente el trípode con la cámara y comprobar que el encuadre fuera el requerido para el estudio. Otra limitación de este estudio fue la iluminación, ya que se generaban sombras en la pared de fondo y hacía difícil la identificación de algunos puntos antropométricos, principalmente el punto "Stomion" en las fotografías de perfil, no obstante, los puntos se podían identificar de todas formas y uno de los objetivos de este trabajo era que el protocolo de fotografía se pudiera repetir fácilmente en otros lugares.

Este trabajo de investigación surgió de la inquietud sobre la fidelidad de la fotogrametría como método de antropometría indirecta. Se basó en la comparación de mediciones faciales realizadas directamente y fotogramétricamente, al igual que los estudios nombrados anteriormente, sin embargo se compararon sólo distancias lineales, no ángulos ni inclinaciones como Farkas, 1980 y Han, 2010. Otra diferencia con los estudios anteriores es que se utilizaron tres objetivos fotográficos de diferentes longitudes para determinar cuál era el que generaba mayor fidelidad en la fotografía facial con respecto a la realidad, y por último, que se dio énfasis al encuadre de la fotografía, ajustando la distancia cámara-sujeto.

\section{CONCLUSIÓN}

Se logró establecer un protocolo con los parámetros de la cámara fotográfica y el encuadre adecuados para obtener, mediante fotogrametría, medidas faciales muy fieles a las medidas directas.

No cualquier cámara ni lente pueden proporcionar una imagen tan cercana a la realidad. Las imágenes obtenidas con lente de $35 \mathrm{~mm}$ se alejan mucho de las medidas reales, y las obtenidas con lente de $50 \mathrm{~mm}$, a pesar de estar más cercanas a la realidad, tampoco logran el objetivo planteado. El método de fotogrametría estandarizada establecida en este trabajo, se basa en la utilización de una cámara réflex con lente fijo de $100 \mathrm{~mm}$, y un encuadre de la imagen de $30 \mathrm{~cm}$.

Este método además es simple y puede ser reproducido fácilmente en cualquier consulta dental.

\section{DECLARACIÓN DE INTERÉS}

Se declara que no existe conflicto de interés por parte de los investigadores ni la existencia de fuentes de financiamiento.

\section{Bibliografía}

1. Han K, Kwon H, Choi T, Kim J, Son D. Comparison of anthropometry with photogrammetry based on a standardized clinical photographic technique using a cephalostat and chair. J Craniomaxillofac Surg. 2010;38(2):96-107.

2. Sheridan P. Practical aspects of clinical photography: Part 1 - Principles, equipment and technique. ANZ J Surg. 2013;83(3):188-191.

3. Farkas L, Bryson W, Klotz J. Is photogrammetry of the face reliable?. Plast Reconstr Surg. 1980;66(3):346-355.

4. Ettorre G, Weber M, Schaaf H, Lowry J, Mommaerts M, Howaldt H. Standards for digital photography in cranio-maxillo-facial surgery - Part I: Basic views and guidelines. J Craniomaxillofac Surg. 2006;34(2):65-73.

5. Schaaf H, Streckbein P, Ettorre G, Lowry J, Mommaerts M, Howaldt H. Standards for digital photography in cranio-maxillo-facial surgery - Part II: Additional picture sets and avoiding common mistakes. J Craniomaxillofac Surg. 2006;34(7):444-455

6. Solow B, Tallgren A. Natural head position in standing subjects. Acta Odontol Scand. 1971;29(5):591-607.

7. Cuccia A, Caradonna C. The natural head position. Different techniques of head positioning in the study of craniocervical posture. Minerva Stomatol. 2009;58:601612.

8. Cuccia A, Carola C. The measurement of craniocervical posture: A simple method to evaluate head position. Int J Pediat Otorhinolaryngol. 2009;73(12):1732-1736.

9. Bister D. Natural head posture--considerations of reproducibility. Eur J Orthod. 2002;24(5):457-470
10. Bister D, Mordarai F, Aveling R. Comparison of 10 digital SLR cameras for orthodontic photography. J Orthod. 2006;33(3):223-230.

11. Aksu M, Kaya D, Kocadereli I. Reliability of reference distances used in photogrammetry. Angle Orthod. 2010;80(4):670-677.

12. Lundstrom A, Lundstrom F, Lebret L, Moorrees C. Natural head position and natural head orientation: basic considerations in cephalometric analysis and research. Eur J Orthod. 1995;17(2):111-120.

13. Madsen D, Sampson W, Townsend G. Craniofacial reference plane variation and natural head position. Eur J Orthod. 2008;30(5):532-540.

14. Peng L, Cooke M. Fifteen-year reproducibility of natural head posture: A longitudinal study. Am J Orthod Dentofacial Orthop. 1999;116(1):82-85

15. Cooke M, Orth D. Five-year reproducibility of natural head posture: A longitudina study. Am J Orthod Dentofacial Orthop. 1990;97(6):489-494.

16. Vernedas A. Iniciación a la fotomacrografía dental. Barcelona: El Vern Edicions; 1998.

17. Davies A. Enciclopedia de la fotografía. Editorial La Isla S.R.L; 2000.

18. Luengo M. La cámara digital. Cómo hacer buenas fotos. San Rafael 4. 28108 Alcobendas Madrid: Editorial LIBSA.; 2005. 